\title{
High "population attributable fraction" for coronary heart disease mortality among relatives in monogenic familial hypercholesterolemia
}

\author{
Melissa A. Austin, PhD ${ }^{1}$, Ron L. Zimmern, FRCP, FFPHM ${ }^{2}$, and Steve E. Humphries, PhD ${ }^{3}$
}

\begin{abstract}
Purpose: To estimate "population attributable fraction" (PAF) for coronary heart disease (CHD) mortality in a population of first-degree relatives of patients with monogenic familial hypercholesterolemia (FH) compared with the PAF for hypercholesterolemia in the general population. Methods: PAF was calculated as $\{f(R-1) /[1+f(R-$ 1)]\}, where $f$ is the frequency of the risk factor (hypercholesterolemia) and $R$ is the relative risk for the association of hypercholesterolemia and CHD death. For FH relatives, f was assumed to be $50 \%$, based on a fully penetrant, dominant mode of inheritance, and $\mathrm{R}$ values were obtained from the prospective Simon Broome Register data. PAFs for hypercholesterolemia and CHD death in the general population were based on the Framingham risk equations for the 95th percentile of cholesterol and CHD mortality. Results: Over all ages, 44\% and 57\% of 5-year CHD mortality could potentially be prevented among male and female first-degree relatives in FH families, respectively, by cholesterol reduction. In contrast, values for 5-year CHD death for hypercholesterolemia in the general population were uniformly lower at all ages, with overall $5 \%$ and $10 \%$ of fatal CHD prevented among men and women, respectively. Conclusion: These results strongly support the view that family based testing strategies of relatives of probands with monogenic hypercholesterolemia, followed by effective lipid lowering drug treatment, is a highly effective way of reducing CHD deaths among these relatives. Genet Med 2002:4(4):275-278.
\end{abstract}

Key Words: coronary heart disease, hypercholesterolemia, population attributable fraction, mortality

The increased risk of coronary heart disease (CHD) in patients with heterozygous familial hypercholesterolemia $(\mathrm{FH})$, the dominant mode of inheritance of this disorder, and the estimated prevalence of $1 / 500$, are well established. ${ }^{1-3}$ Monogenic FH is diagnosed clinically based on elevated total cholesterol concentrations and the presence of tendon xanthomas and family history of hyperlipidemia and premature $\mathrm{CHD}^{4}$ or on the basis of low-density lipoprotein receptor gene mutations. ${ }^{5,6} \mathrm{~A}$ recent analysis demonstrated that family testing for FH among first-degree relatives is cost-effective in the United Kingdom ${ }^{7}$ and ongoing family testing in the Netherlands has dramatically increased the percentage of adults receiving lipidlowering treatment. ${ }^{8}$

From a public health perspective, the population attributable fraction (PAF) can be used to evaluate and compare the impact of risk factors on disease risk. PAF is defined as the

From the ${ }^{1}$ Institute for Public Health Genetics and Department of Epidemiology, School of
Public Health and Community Medicine, University of Washington, Seattle, Washington;
${ }^{2}$ Public Health Genetics Unit, Strangeways Research Laboratory, University of Cambridge,
Cambridge, United Kingdom; and ${ }^{3}$ Center for Genetics of Cardiovascular Disorders, British
Heart Foundation Laboratories, Department of Medicine, Royal Free and University College
London Medical School, London, United Kingdom.

Melissa A. Austin, PhD, Institute for Public Health Genetics, Box 357236, 1959 NE Pacific

Avenue, University of Washington, Seattle, WA 98195-7236.

Received: January 2, 2002.

Accepted: March 26, 2002.

DOI: 10.1097/01.GIM.0000020749.39075.2A proportion of cases of disease in a population that is attributable to a specific risk factor and, thus, estimates the proportion of disease that could be prevented if that risk factor were eliminated. $^{9-11}$ To our knowledge, this approach has not been applied to relatives in families with monogenic disorders. ${ }^{12}$ The term PAF is normally calculated by reference to a general population base, but in this analysis, we define the "population" at risk to be first-degree relatives of patients with $\mathrm{FH}$ and calculate the PAF for that specific population. In a monogenic condition such as FH, the frequency of "exposure" can be accurately estimated from the known, dominant, mode of inheritance. Furthermore, there is nearly full penetrance of this genetic disorder, consistently resulting in hypercholesterolemia among relatives with FH mutations. Importantly, effective therapy to reduce risk of disease, namely statin treatment, is widely available to reduce risk of CHD. ${ }^{13-15}$ Thus the aim of this analysis was to estimate PAF for FH and CHD mortality among first-degree relatives in $\mathrm{FH}$ families, assuming that CHD mortality is caused by hypercholesterolemia, and to compare these findings with general population-based estimates for hypercholesterolemia.

\section{METHODS}

Determination of PAF requires estimates of the frequency of the risk factor in the population of interest ( $\mathrm{f}$ ) and the relative 
risk for the association of that risk factor with disease (R), and is calculated as $\mathrm{PAF}=\{\mathrm{f}(\mathrm{R}-1) /[1+\mathrm{f}(\mathrm{R}-1)]\} .^{9-11}$

For FH families, the "population" consisted of first-degree relatives (parents, siblings, and offspring) of patients with FH. Assuming a dominant mode of inheritance, full penetrance, and no phenocopies (polygenic hypercholesterolemia), 50\% of such relatives would be "exposed" to an FH mutation, resulting in $\mathrm{f}_{\mathrm{FH}}=0.50$. Relative risks for the association between $\mathrm{FH}$ and coronary heart disease mortality $\left(\mathrm{R}_{\mathrm{FH}}\right)$ were based on prospective data from the Simon Broome Register using 20-year age groups by sex for 5-year risk of CHD mortality ${ }^{2}$ (Table 1 ). Because $\mathrm{f}_{\mathrm{FH}}$ was held constant at 0.50 in this analysis, $95 \%$ confidence intervals for PAF were calculated to correspond to the $95 \%$ confidence limits for $\mathrm{R}_{\mathrm{FH}}$.

PAFs for hypercholesterolemia and CHD were determined for the general population, based on data from the Framingham Offspring Study. ${ }^{16}$ The Framingham risk functions were used to estimate 5-year CHD death rates for men and women by 10 -year age groups, assuming subjects were nonsmoking, did not have diabetes, and did not have left ventricular hypertrophy. ${ }^{17}$ The relative risks for hypercholesterolemia and CHD death were calculated for each age/sex group by taking the ratio of absolute risk for total cholesterol at 95th percentile compared with the absolute risk for total cholesterol at median value for each sex/age group. Thus, by definition, the frequency of hypercholesterolemia was $5 \%$ in each group. The 95th percentile values for the age/sex groups were generally slightly lower than the value of $7.5 \mathrm{mmol} / \mathrm{L}$ used to define $\mathrm{FH}$ clinically. ${ }^{4}$

\section{RESULTS}

As shown in Table 1, most of the 5 -year CHD mortality is preventable among male and female first-degree relatives under age 40 in $\mathrm{FH}$ families by treating hypercholesterolemia in

\section{Table 1}

PAF for 5-year CHD mortality among first-degree relatives of probands with monogenic FH, by sex and age

\begin{tabular}{lccl}
\hline Sex & $\begin{array}{c}\text { Age group } \\
(\mathrm{yr})\end{array}$ & $\mathrm{R}_{\mathrm{FH}}(95 \% \mathrm{CI})^{a}$ & $\begin{array}{c}\text { PAF in FH first-degree } \\
\text { relatives }(95 \% \mathrm{CI})\end{array}$ \\
\hline Males & $20-39$ & $48.4(17.8,105.5)$ & $0.96(0.89,0.98)$ \\
& $40-59$ & $3.5(1.9,6.0)$ & $0.56(0.31,0.71)$ \\
& $60-79$ & $1.1(0.5,2.3)$ & $0.05(-0.33,0.39)$ \\
& $20-79$ & $2.6(1.7,3.8)$ & $0.44(0.26,0.58)$
\end{tabular}

\begin{tabular}{lccc} 
Females & $20-39$ & $125.0(15.1,451.3)$ & $0.98(0.88,1.00)$ \\
& $40-59$ & $8.4(3.1,18.4)$ & $0.79(0.51,0.90)$ \\
& $60-79$ & $2.6(1.3,4.5)$ & $0.44(0.13,0.64)$ \\
& $20-79$ & $3.7(2.3,5.8)$ & $0.57(0.39,0.71)$ \\
\hline
\end{tabular}

\footnotetext{
${ }^{a}$ Based on prospective data from the Simon Broome Register ${ }^{2}$; CI, confidence interval; assumes $\mathrm{f}_{\mathrm{FH}}$ (frequency of $\mathrm{FH}$ among first-degree relatives in families with an $\mathrm{FH}$ proband) $=0.5$.
}

these groups ( $\mathrm{PAF}=0.96$ and 0.98 , respectively). Although the confidence intervals for $\mathrm{R}_{\mathrm{FH}}$ in these groups were wide, the PAFs corresponding to the lower bound of the confidence intervals were still high: 0.89 and 0.88 for males and females, respectively. For male first-degree relatives ages 40 to 59 years, approximately half of the CHD mortality could be prevented, whereas the PAF decreases to approximately $5 \%$ for the older age group due to the low relative risk $\left(\mathrm{R}_{\mathrm{HC}}=1.1\right)$. Among female first-degree relatives, the PAF is approximately 0.75 in the 40 to 59 year age group and remains at least one-third in the oldest age group. Over all ages, between $44 \%$ and $57 \%$ of CHD mortality could potentially be prevented among male and female first-degree relatives in FH families, respectively, by treating hypercholesterolemia among affected relatives. These estimates are not materially altered by small changes in the estimated frequency of detectable FH based on differences in response rate and number of affected subjects, as observed in a pilot study in Manchester ( $\mathrm{f}_{\mathrm{FH}}$ ranging from 0.36 to $0.60^{18}$ ).

As shown in Figure 1, with the exception of men aged 60 years and older, the population-based PAF values for hypercholesterolaemia and 5-year CHD death were dramatically lower than the values for first-degree relatives in $\mathrm{FH}$ families for all age/sex groups. As with the findings for FH, the PAF values decreased with age and were generally higher for men than women. Combining all ages, approximately $5 \%$ and $10 \%$ of fatal CHD is preventable among men and women, respectively, from the general population, by reducing hypercholesterolemia. The Framingham-based calculations were repeated using the 98th percentile for each sex/age group (cut points ranging from 8.80 to $10.1 \mathrm{mmol} / \mathrm{L}$ ), resulting in very similar PAF values (data not shown). These findings are in contrast to the $40 \%$ to $60 \%$ of potentially preventable CHD deaths among first-degree relatives in FH families.

\section{DISCUSSION}

Reducing the mortality and morbidity for $\mathrm{CHD}$ is a major aim of the WHO and many governments worldwide. ${ }^{19,20}$ The results from this analysis clearly demonstrate that cascade testing provides major health benefits for relatives of patients with $\mathrm{FH}$. The risk of untreated FH and CHD death is uniformly high, especially in younger age groups (Table 1). ${ }^{2}$ Statins are effective treatments, ${ }^{21}$ and cascade testing will allow the categorization of the relatives into those with the $\mathrm{FH}$ mutation and those without. By contrast, findings based on Framingham risk equations suggest that the identification and management of hypercholesterolemia, in the absence of other risk factors, will not have a major effect on cardiovascular mortality in the general population (Fig. 1). However, because of the low prevalence of FH mutations ( 1 in 500), the identification and treatment of all FH cases would be unlikely to have a significant impact on cardiovascular mortality in the general population.

In this analysis, we applied the public health concept of "population attributable fraction" to estimate the proportion of CHD deaths preventable by reduction in cholesterol levels among relatives in FH families. The FH mutation, unlike usual 


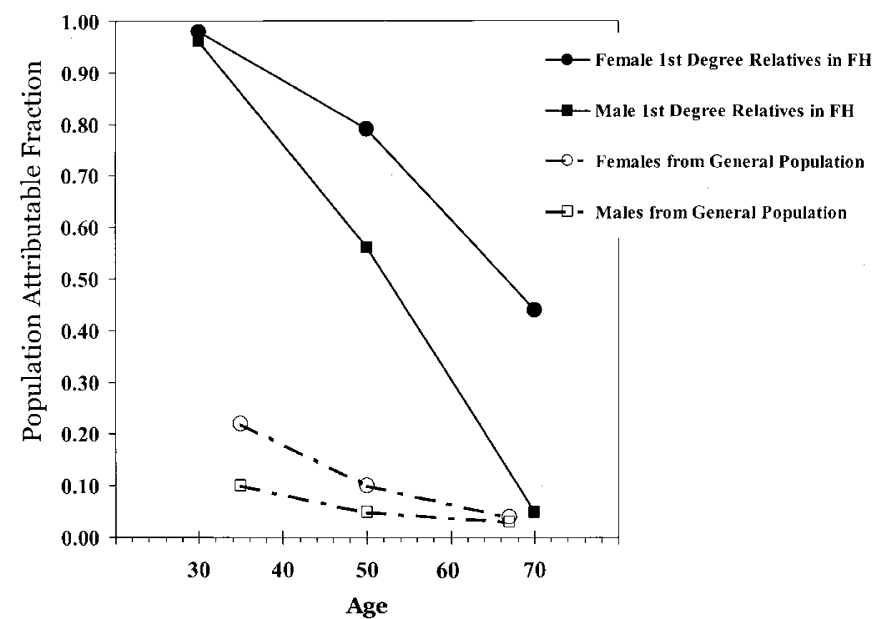

Fig. 1 PAF estimates for cholesterol reduction and CHD mortality by sex and age among first-degree relatives of patients with monogenic $\mathrm{FH}$ (solid lines) and for the general population (dashed lines). Median ages are plotted for age groups. Table 1 provides relative risk values for CHD mortality in FH families. Relative risks for cholesterol over the 95th percentile and CHD mortality in the general population, based on Framingham risk equations, ${ }^{16,17}$ were $3.28,2.14$, and 1.66 for males in age groups 30 to 39,40 to 59 , and 60 to 74 , respectively; relative risks for females were $6.67,3.06$, and 1.75 for the same age groups.

environmental exposures for which PAFs are calculated, cannot be eliminated, but the penetrance of the risk factor consequent to the exposure, hypercholesterolemia, can be significantly reduced with pharmacological therapy and can lead to a significant reduction in mortality. The PAF, therefore, represents the theoretical limits of prevention effectiveness in this population group that may be attributed to a reduction in cholesterol levels. The results demonstrate that nearly all CHD deaths could be prevented under age 40 and more than half of such deaths could be prevented between ages 40 and 59. By contrast, using Framingham data, ${ }^{16,17}$ the proportion of CHD deaths that could be prevented in the general population ranged from 0.22 to 0.05 in similar sex and age groups.

In the general population, hypercholesterolemia is caused by the coinheritance of several genetic factors (polygenic) interacting with environmental factors (e.g., diet). Even though first-degree relatives of such individuals are likely to have inherited a certain degree of genetic predisposition to hypercholesterolemia, testing their relatives is unlikely to be a cost-effective way of identifying further subjects with severely elevated plasma cholesterol levels. By contrast, for monogenic $\mathrm{FH}$, the proportion of first-degree relatives who have inherited the genetic predisposition is $50 \%$, due to the known dominant mode of inheritance. Many, if not all, of these carriers will have been exposed to an excess burden of cholesterol since birth or early childhood. This makes the testing of relatives of a proband a highly efficient strategy to identify at-risk subjects in these families.

The actual number of first-degree relatives with $\mathrm{FH}$ that could be identified using such a strategy clearly depends on family size. However, each identified subject is then a potential new proband for further testing of his or her first-degree rela- tives. Such a sequential sampling strategy should be a highly efficient way to identify at-risk subjects, dependent only on family size and loss due to nonresponse. Such an approach for FH has been used by the MedPed organization ${ }^{22}$ and shown to be effective in the U.K. and Holland.8,18 Family based approaches raise issues of confidentiality and presymptomatic testing causes anxiety, but the effect of $\mathrm{FH}$ testing has been reported to be minimal and not long lasting, ${ }^{23-26}$ and the health benefits are substantial.

There are several assumptions and limitations of this analysis. First, although risk in the general population was estimated from the Framingham Offspring Study, these risk functions have been shown to be valid for a variety of population groups. ${ }^{27,28}$ For $\mathrm{FH}$, risk estimates are based on the largest study published to date, ${ }^{2}$ but they still only represent a total of only 46 deaths from CHD, and the confidence intervals on these estimates are wide (Table 1). Second, the available data may overestimate or underestimate the true risk effect. The estimates are based on previous studies where ascertainment bias and incomplete data may have occurred and the subjects recruited may overrepresent those most severely affected so that the actual relative risk in FH subjects in the general population may be lower. Alternatively, because subjects in the Simon Broome register were being treated with resins, dietary advice, etc., which, although of small effect on lipid lowering compared with the statins, would still have reduced the relative risk estimate of "untreated FH." However, because it is unethical to withhold treatment for $\mathrm{FH}$, there are few studies where this risk can be determined. Third, full penetrance of FH has been assumed. Because clinical endpoints of $\mathrm{FH}$ may vary within families, and the apolipoprotein B Arg3500Gln mutation $^{29}$ or the autosomal recessive form of $\mathrm{FH}^{30}$ could be present among FH families, this assumption may not be appropriate. Fourth, it is assumed that cholesterol reduction using statins will reduce the risk of CHD mortality in patients with $\mathrm{FH}$ to a similar extent as the reduction seen in the general population. Although statins clearly do reduce mortality in $\mathrm{FH},{ }^{3}$ it is unknown if complete reduction of risk is possible.

Fifth, the population attributable fraction was calculated only for CHD mortality because morbidity information is not reliably available for any large group of $\mathrm{FH}$ patients. Importantly, the attributable fraction calculation assumes a causal relationship between the risk factor and the disease, i.e., reduction in the penetrance of hypercholesterolemia caused by $\mathrm{FH}$ mutations, will reduce CHD death. As a result, the PAFs values may be overestimates, but at least they reflect a reduction in risk toward the level of the general population. There is still considerable debate as to what the optimum "target" level of plasma lipids is in subjects with hypercholesterolemia, and whether there is a continued benefit in lower risk for lower cholesterol by increasing the dose of statins. ${ }^{31}$ Once again, cost-benefit considerations are relevant because the cost of statin therapy is not inconsiderable (roughly $\mathfrak{E} 430$ per year including GP time ${ }^{7}$ ) and higher doses increases the risk of side effects. 
Finally, this analysis has not evaluated the potential effects of gene-gene interactions, gene-environment interactions, or the confounding effects of other risk factors. For example, non-insulin-dependent diabetes is a major risk factor for CHD events, the prevalence of diabetes increases with age, and would be likely to alter PAF estimates. However, it has been shown recently that statin treatment reduces CHD events in diabetics, ${ }^{32}$ further strengthening the benefit of this therapeutic approach.

Despite these considerations, the population attributable fraction estimates presented here strongly support the view that family-based testing strategies of relatives of probands with monogenic hypercholesterolemia, including FH mutations, followed by effective lipid lowering drug treatment, is a highly effective clinical intervention to reduce CHD deaths among families with FH. Furthermore, because dietary and lifestyle changes are also an important part of risk reduction for $\mathrm{FH}$, a family based approach has considerable advantages. We believe that its use should be strongly encouraged as a matter of good clinical practice.

The case for cascade testing is based on clinical and not on public health considerations, because low prevalence of $\mathrm{FH}$ limits the public health impact of intervention. Cardiologists, and physicians who manage these patients must be aware of the benefits of identifying and treating patients with $\mathrm{FH}$ through cascade testing, and providers of cardiac services should ensure that resources are made available to enable widespread implementation of this effective clinical intervention.

\section{Acknowledgments}

Dr. Humphries is funded by the British Heart Foundation (RG2000015, RG93008). This work was supported by University Initiative Fund of the University of Washington and NIH grants HL49513 from NHLBI and P30ES07033 from NIEHS, and the UW Center for Genomics and Public Health (ASPH/ CDC/ATSDR S1946-21/21). The study was performed during Dr. Austin's sabbatical as Visiting Professor at University College London and Honorary Visiting Fellow at the Public Health Genetics Unit, University of Cambridge. The authors thank Emma Hawe, Aroon Hingorani, and Dr. Stephen Walter for their valuable technical assistance.

\section{References}

1. Goldstein JL, Hobbs HH, Brown MS. Familial hypercholesterolemia. In: Scriver CR, Beaudet AL, Sly WS, Valle D, editors. The metabolic basis of inherited disease. New York: McGraw Hill, 1995:1981-2030.

2. Scientific Steering Committee on behalf of the Simon Broome Register Group. Mortality in treated heterozygous familial hypercholesterolemia: implications for clinical management. Atherosclerosis 1999;142:105-112.

3. Simon Broome Steering Committee. Risk of fatal coronary heart disease in familial hypercholesterolemia. Scientific Steering Committee on behalf of the Simon Broome Register Group. BMJ 1991;303:893-896.

4. Neil HAW, Hammond T, Huxley R, Matthews DR, Humphries SE. Extent of underdiagnosis of familial hypercholesterolemia in routine practice: prospective registry study. BMJ 2000;321:148.

5. Day INM, Whittall RA, O'Dell SD, Haddad L, Bolla MK, Gudnason V, Humphries SE. Spectrum of LDL receptor gene mutations in heterozygous familial hypercholesterolemia. Hum Mutat 1997;10:116-127.
6. Heath KE, Humphries SE, Middleton-Price H, Boxer M. A molecular genetic service for diagnosing individuals with familial hypercholesterolaemia (FH). Eur J Hum Gen 2001;9:244-252.

7. Marks D, Wonderling D, Thorogood M, Lambert H, Humphries SE, Neil HAW Screening for hypercholesterolaemia versus case finding for familial hypercholesterolaemia: a systematic review and cost-effectiveness analysis. Health Technol Assess 2000;4:1-123.

8. Umans-Eckenhausen MAW, Defesche JC, Sijbrands EJG, Scheerder RLMJ, Kastelein JJ. Review of the first 5 years of screening for familial hypercholesterolemia in the Netherlands. Lancet 2001;357:165-168.

9. Levin MS. The occurrence of lung cancer in man. Acta Unio Int Contra Cancrum 1953;9:531-541.

10. Hennekens CH, Buring JE. Attributable risk. In: Mayrent SL, Doll SR, editors. Epidemiology in medicine. Boston: Little, Brown and Company, 1987:87-95.

11. Khoury MJ, Beaty TH, Cohen BH. Attributable fraction. In: Fundamentals of genetic epidemiology. New York: Oxford University Press, 1993:77-79.

12. Krawczak M, Cooper DN, Schmidtke J. Estimating the efficacy and efficiency of cascade genetic screening. Am J Hum Genet 2001;69:361-370.

13. Shepherd J, Cobbe SM, Ford I, Isles CG, Lorimer AR, MacFarlane PW, McKillop JH, Packard CJ. Prevention of coronary heart disease with pravastatin in men with hypercholesterolemia. West of Scotland Coronary Prevention Study. N Engl J Med 1995;333:1301-1307.

14. Smilde TJ, van Wissen S, Wollersheim H, Trip MD, Kastelein JJP, Stalenhoef AFH. Effect of aggressive versus conventional lipid lowering on atherosclerosis progression in familial hypercholesterolaemia (ASAP): a prospective, randomised, doubleblind trial. Lancet 2001;357:577-581.

15. Corti R, Fayad ZA, Fuster V, Worthley SG, Helft G, Chesebro J, Mercuri M, Badimon JJ. Effects of lipid-lowering by simvastatin on human atherosclerotic lesions. A longitudinal study by high-resolution, noninvasive magnetic resonance imaging. Circulation 2001;104:249-252.

16. Anderson KM, Wilson PWF, Odell PM, Kannel WB. An updated coronary risk profile. A statement for health professionals. Circulation 1991;83:356-362.

17. Hingorani AD, Vallance P. A simple computer program for guiding management of cardiovascular risk factors and prescribing. BMJ 1999;318:101-105.

18. Bhatnagar D, Morgan J, Siddiq S, Mackness MI, Miller JP, Durrington PN. Outcome of case finding among relatives of patients with known heterozygouts familial hypercholesterolemia. BMJ 2000;321:1-5.

19. Wood D. Established and emerging cardiovascular risk factors. Am Heart J 2001; 141:S49-S57.

20. Executive summary of the third report of The National Cholesterol Education Program (NCEP) Expert Panel on Detection, Evaluation, and Treatment of High Blood Cholesterol in Adults (Adult Treatment Panel III). JAMA 2001;285:2486-2497.

21. Randomised trial of cholesterol lowering in 4444 patients with coronary heart disease: the Scandinavian Simvastatin Survival Study (4S). Lancet 1994;344:1383-1389.

22. Familial hypercholesterolemia. Report of a second WHO consultation in Geneva. World Health Organization, Human Genetics Program, Geneva, October, 1999.

23. Tonstad S. Familial hypercholerolaemia: a pilot study of parents and childrens concerns. Acta Pediatr 1996;85:1307-1313.

24. Tonstad S, Novik TS, Vandvik IH. Psychosocial function during treatment for familial hypercholesterolaemia. Pediatrics 1996;98:249-255.

25. Rosenthal SL, Knauer-Black S, Stahl MP, Catalanotto TJ, Sprecher DL. The psychological functioning of children with hypercholesterolemia and their families. Clin Pediatr (Phila) 1993;32:135-141.

26. Humphries SE, Galton D, Nicholls P. Genetic testing for familial hypercholesterolaemia: practical and ethical issues. Q J Med 1997;90:169-181.

27. Haq IU, Ramsay LE, Yeo WW, Jackson PR, Wallis EJ. Is the Framingham risk function valid for northern European populations? A comparison of methods for estimating absolute coronary risk in high risk men. Heart 1999;81:40-46.

28. D'Agostino RBS, Grundy S, Sullivan LM, Wilson P, for the CHD Risk Prediction Group. Validation of the Framingham coronary heart disease prediction score. Results of a multiple ethic groups investigation. JAMA 2001;286:180-187.

29. Tybjaerg-Hansen A, Steffensen R, Meinertz H, Schnohr P, Nordestgaard BG. Association of mutations in the apolipoprotein $\mathrm{B}$ gene with hypercholesterolemia and the risk of ischemic heart disease. N Engl J Med 1998;338:1577-1584.

30. Garcia CK, Wilund K, Arca M, Zuliani G, Fellin R, Maioli M, Calandra S, Bertolini S, Cossu F, Grishin N, Barnes R, Cohen JC, Hobbs HH. Autosomal recessive hypercholesterolemia caused by mutations in a putative LDL receptor adaptor protein. Science 2001;292:1394-1398.

31. Thompson GR. What targets should lipid-modulating therapy achieve to optimise the prevention of coronary heart disease? Atherosclerosis 1997;131:1-5.

32. Freeman DJ, Norrie J, Sattar N, Neely RD, Cobbe SM, Ford I, Isles C, Lorimer AR, Macfarlane PW, McKillop JH, Packard CJ, Shepherd J, Gaw A. Pravastatin and the development of diabetes mellitus: evidence for a protective treatment effect in the West of Scotland Coronary Prevention Study. Circulation 2001;103:357-362. 